\title{
INVESTIGACIÓN ETNOGRÁFICA EN AMBIENTES DIGITALES: UNA EXPERIENCIA METODOLÓGICA EN DOS PROYECTOS DE DOCTORADO EN EL CONTEXTO DE LA EDUCACIÓN SUPERIOR EN COLOMBIA
}

\section{Ethnographic investigation in digital atmospheres: a methodological experience in two doctorate projects in the context of the higher education in Colombia}

\author{
Julio Ernesto Rojas Mesa* \\ Carlos Bernal Granados**
}

Recibido: 6 de marzo de 2008 • Revisado: 25 de abril de 2008 • Aceptado: 23 de mayo de 2008

\begin{abstract}
Resumen
Nos abocamos a la necesidad de pensar, con más intensidad y variedad, la relación virtualidad-educación superior en Colombia. Los debates al respecto son numerosos, las posiciones teóricas son variadas, los lugares desde los cuales se analiza la situación son igualmente múltiples. Las dos investigaciones presentadas aquí, pretenden establecer un análisis de tal relación, localizando dicho análisis en varias instituciones de educación superior en Colombia. La primera investigación, titulada Educación virtual: del discurso a las prácticas pedagógicas. Un estudio etnográfico en seis instituciones de Educación Superior en Colombia, pretende identificar sobre la base del estudio etnográfico, la interpretación de la relación entre el mito/discurso, el rito/práctica y el territorio/escenario, que se construyen en las instituciones educativas seleccionadas para el estudio en torno a la educación virtual. La segunda investigación, titulada: Fundamentación teórica de la educación virtual en la educación superior en Colombia, se desarrolla sobre el enfoque semiótico de búsqueda del estatuto teórico
\end{abstract}

Candidato a Doctor en Teoría de la Educación y Pedagogía Social. UNED-España. Diplomado en Estudios Avanzados en Teoría de la Educación y Pedagogía Social. Antropólogo. Universidad Nacional de Colombia. Docente Departamento de Humanidades. Universidad Santo Tomás. Correo electrónico: juliorojasmesa@yahoo.com.

** Candidato a Doctor en Teoría de la Educación y Pedagogía Social. UNED-España. Magister en Literatura Latinoamericana. Especialista en Informática y Multimedios; Multimedia Creativa y, en Gestión y Evaluación Curricular. Diplomado en Estudios Avanzados en Teoría de la Educación y Pedagogía Social. Docente de la Facultad de Filosofía de la Universidad Santo Tomás. Correo electrónico: carlosbernalgranados@gmail.com. 
de la educación virtual, teniendo en cuenta cuatro vectores de análisis: lo filosófico, lo comunicativo, lo pedagógico y lo tecnológico. Lo anterior obliga a pensar el estatuto de la investigación en Ciencias Sociales, y sus transformaciones metodológico-académicas contemporáneas. La etnografía da cuenta de dicho proceso en su propia transformación metodológica.

\title{
Palabras clave
}

Etnografía, etnografía virtual, educación virtual, metodologías cualitativas, ambientes digitales, Internet, cibercultura, comunidades virtuales, antropología, semiótica.

\begin{abstract}
In this project, the researchers find the necessity of thinking about the relationship between virtual education and university education in Colombia at a deeper and more diverse level. There have been a great number of debates about the topic, a variety of theoretical positions and multiple places where this situation has been analyzed from. The two researches described in this paper are aimed to establish an analysis of such relationship in several university education institutions in Colombia. The purpose of the first research, Virtual Education: From the Discourse to the Pedagogical Practices. An Ethnographic Study in Six University Institutions in Colombia, is to identify, on the basis of an ethnographic study, the interpretation of the relationship between myth/ discourse, rite/practice and territory/scenario that is built up in the education institutions chosen for carrying out the research about virtual education. The second research, Theoretical Foundations of Virtual Education in University Education in Colombia is developed on the semiotic approach for finding the virtual education theoretical statute taking into consideration four analysis vectors: the philosophical, communicative, pedagogical and technological vectors. This creates the necessity of studying the research statute for social sciences and its contemporary methodological/academic transformations under influence. The ethnography shows that process in its own methodological transformation.
\end{abstract}

\section{Key Words}

Ethnography, on-line ethnography, on-line education, qualitative methodologies, digital environments, Internet, cyber culture, on-line communities, anthropology, semiotics.

\section{Introducción}

La estructuración, desarrollo y aplicación metodológica del presente trabajo de campo, se inscribe dentro de los estudios de doctorado en Teoría de la Educación y Pedagogía Social, realizados en la Universidad Nacional de Educación a Distancia: UNED, con sede en Madrid-España, de los proyectos de tesis doctoral de los autores de este artículo.

De acuerdo con los requerimientos académicos necesarios, en relación con la socialización a la comunidad académica de los avances de las tesis doctorales, presentamos este informe cuyo contenido se enfoca en el diseño y justificación de la propuesta metodológica realizada para el desarrollo de dichos trabajos de investigación.

El informe contiene los elementos necesarios para identificar los intereses teóricos particulares de cada investigación y también para el cumplimiento de los objetivos propuestos en cada una de ellas, cuyos intereses temáticos comparten en común, el tema 
de la educación virtual en la educación superior en Colombia'.

\section{Antecedentes del contexto etnográfico de la investigación}

Indudablemente, el marco antropológico, a partir del cual arrancamos esta reflexión, está relacionado con las diferentes crisis vividas por la Antropología, la crisis del objeto, del método y de la representación. La crisis del objeto es evidenciada inicialmente por la incredulidad sobre la supuesta objetividad de los estudios etnográficos, lo cual evidencia que en cada postura teórica hay una posición cultural (Vasco, 1987). Situación confirmada de manera contundente con la aparición de la nueva etnografía y la visión émica del trabajo etnográfico (visión del sujeto investigado). En relación con la crisis del método, Velasco y Rada, realizarán el siguiente comentario:

La crisis de método planteada desde la corriente de la nueva etnografía, descubrió como exigencia el conocimiento de una cultura para poder formular preguntas significativas a los informantes, Ya no podría decirse que se había logrado el conocimiento de una cultura cuando se supieran de antemano las respuestas de los informantes, sino cuando el investigador fuera capaz de realizar preguntas significativas. La meta de la investigación paso a ser el estudio de los códigos culturales (1997, p. 74).

La otra gran crisis (de la representación) está situada en el estatuto epistemológico de la disciplina como tal. A partir del siglo XIX se vislumbra la necesaria transformación de la perspectiva del hombre como centro del universo, premisa sostenida durante mucho tiempo por la Modernidad y que en los estudios

1 La metodología propuesta para la investigación de campo conjunta, por una parte, se propuso con miras a compartir esfuerzos a partir de la experiencia de cada investigador, y también, por el hecho de continuar el trabajo colaborativo que los dos investigadores que desde el punto de vista académico han sostenido desde el mismo inicio del Doctorado. Por otra parte, por la motivación y común acuerdo por parte de la directora de las dos investigaciones. Doctora Marta Ruíz Corbella. contemporáneos, Michel Foucault develaría de manera sistemática (Foucault, 1971).

Quizá habría que ver el primer esfuerzo por lograr este desarraigo de la Antropología, al que sin duda está consagrado el pensamiento contemporáneo, en la experiencia de Nietzsche: a través de una crítica filológica, a través de cierta forma de biologismo, Nietzsche encontró de nuevo el punto en el que Dios y el hombre se pertenecen uno a otro, en el que la muerte del segundo es sinónimo de la desaparición del primero y en el que la promesa del superhombre significa primero $y$ antes que nada, la inminencia de la muerte del hombre (p. 31).

Hablar entonces del método etnográfico en la investigación social, significa plantearse la pregunta por la validez, y conmensurabilidad del grupo de estudio, nótese que ha quedado atrás la definición de éste como objeto, ha quedado atrás la herencia y validez de la emulación del método científico de las ciencias exactas en la investigación social, y por último, ha quedado atrás el estatuto epistemológico de la antropología como saber equivalente a la ontología del ser en el mundo, planteado por Kant en la Modernidad:

Esto había sido formulado ya por Kant en la lógica al agregar, una última interrogación a su trilogía tradicional: las tres preguntas críticas (¿que puedo saber?, ¿Qué debo hacer?, ¿Qué me es permitido esperar?), están relacionadas, pues con una cuarta y en cierta forma, dependen de ella ¿Qué es el hombre? (Foucault, 1971, p. 331).

Tradicionalmente, el método etnográfico ha tenido un lugar ambivalente y relativo, en el análisis de las culturas y sociedades; desde muy temprano, la manera como se estudiaba a las poblaciones consideradas primitivas para recoger información que sirviera para entender los procesos sociales de las sociedades autodenominadas "civilizadas", fue un proceso diverso y heterogéneo. Atkinson y Hammersley (1994), en el texto elaborado sobre Etnografía y métodos de investigación, describe algunos de los escenarios y accionar de este campo de conocimiento: 
Así, a lo largo de los numerosos campos en que ha sido propuesta la etnografía, o a veces algo parecido a ella, podemos encontrar diferencias considerables en cuanto a las prescripciones y a la propia práctica. Existe desacuerdo sobre si la característica distintiva de la etnografía es el registro del conocimiento cultural (Spradley, 1980), la investigación detallada de padrones de interacción social (Gumperz, 1981) o el análisis holístico de sociedades (Lutz, 1981). Algunas veces la etnografía se define como esencialmente descriptiva, otras veces como una forma de registrar narrativas orales (Walker, 1981); como contraste, sólo ocasionalmente se pone el énfasis en el desarrollo y verificación de teorías (Glaser y Strauss, 1967; Denzin, 1978) (p. 6).

El trabajo de campo da forma a la etnografía, entendido como un ejercicio de interacción directa y prolongada con los sujetos estudiados y en cual Malinowski, (1948), marca un punto importante al establecerlo como una técnica de recolección de información, sistemática y enmarcada en un escenario de observación e interpretación, realizado dentro de los grupos estudiados. Con ello se desbordaba la práctica tradicional de los metalenguajes construidos sobre relatos de comerciantes, funcionarios y otros individuos, práctica en la que se habían basado los antropólogos antecesores, como era el caso de James Frazer y otros. Frazer irrumpe de manera determinante, desde el ámbito antropológico, todo el universo de las disciplinas sociales.

La inclinación de Frazer a considerar los hechos antropológicos como parte integrante de la vida humana en general, dentro del conjunto de la cultura y aún sobre el fondo del paisaje y el ambiente natural, aparece ya, bella y claramente en su comentarios a la traducción de la descripción de Grecia por Pausanias [...] (Malinowski, 1948, p. 219).

Como respuesta, el contexto se erigió como uno de los conceptos clave en la forma malinowskiana y antropológica hasta la fecha, de abordar la etnografía: $E I$ punto de vista antropológico implica, por una parte, la consideración de cada cultura dentro de su ambiente natural (Malinowski, 1948, p. 29).
La recuperación de lo émico dentro del ejercicio etnográfico, pasará a ser un elemento fundamental en la idea del yo y del otro, a la hora de construir representaciones sobre los grupos humanos. En este enfoque de la "Nueva etnografía", respaldado en el replanteamiento de los análisis estructuralistas de los sistemas culturales, Kenet Pike y Charles Frake (Pike y Frake en Harris, 1985), entre otros, comienzan a defender el punto de vista de los miembros del sistema cultural analizado, ello va a ser realmente importante en las construcciones contemporáneas del método etnográfico y de su aplicación en los análisis sociales y culturales de las sociedades contemporáneas.

Una etnografía debería ser una teoría de la conducta cultural en una sociedad dada [...] La prueba de adecuación descriptiva debe estar referida siempre a la interpretación que el informante hace de los acontecimientos, y no simplemente de la ocurrencia de esos acontecimientos (Harris, 1985, p. 29).

Luego, llegará el materialismo cultural aportando un noción de interacción intima entre cultura y entorno físico que hoy día podríamos denominarlo contexto. Sin embargo, aun estaban absolutamente universalizados y se continuaban buscando leyes de la cultura a través del método etnográfico:

La evolución multilineal es esencialmente una metodología basada en la suposición de que el cambio cultural se da en regularidades significativas y orientadas a la determinación de leyes culturales (Harris, 1985, p. 568)

La última época de la Antropología estará enmarcada dentro de dos corrientes teóricas que, en cierta forma, confrontan el estatuto epistemológico y el sentido ideológico de las disciplina como tal. El relativismo cultural de Franz Boas (1948, citado por Harris, 1995) retomado por Cliford Geertz (1997). El enfoque etnográfico que se trabaja desde esta perspectiva no busca determinar leyes o teorías generales que expliquen lo particular y lo universal dentro y fuera de un sistema cultural específico, más bien, se pretende realizar un abordaje hermenéutico e interpretativo que permita identificar horizontes de sentido, construidos desde 
el lenguaje, la práctica y el territorio donde habitan las representaciones de las comunidades estudiadas.

Creyendo con Max Weber, que el hombre es un animal inserto en tramas de significación que el mismo ha tejido, considero que la cultura es esa urdimbre y que el análisis de la cultura ha de ser por tanto, no una ciencia experimental en busca de leyes, sino una ciencia interpretativa en busca de significaciones (Geertz. 1997: 20).

Esta perspectiva, materializada en la corriente de los estudios culturales, tuvo eco en América Latina desde la década del noventa, en el trabajo de Néstor García Canclini, (1990), Beatriz Sarlo, (1994), Renato Ortiz, (1997) y Jesús Martin-Barbero, (2002) entre otros, llegando incluso, en el ámbito académico colombiano, a determinar el enfoque teórico de las carreras de Antropología constituidas por distintas universidades en dicha década y en los años posteriores².

Es igualmente importante referir de manera breve, el contexto de las teorías poscoloniales, por cuanto han llevado a realizar una crítica del relativismo cultural y de la cultura como respuesta. Herederos de la escuela marxista, abogan por una categoría supra-local que defiende lo local: "el Latinoamericanismo". Santiago Castro (1998) describe esta corriente así:

Las teorías poscoloniales se articulan, en cambio, al interior de contextos postradicionales de acción, es decir, en localidades donde los sujetos sociales configuran su identidad interactuando con procesos de racionalización global y donde, por lo mismo, las fronteras culturales empiezan a volverse borrosas. Esto explica en parte por qué teóricos como Said, Bhabha y Spivak no se ven a sí mismos como profetas que articulan la voz del oprimido, como "guardianes" de ninguna tradición cultural extraoccidental o como representantes intelectuales del "tercer mundo". Como veremos enseguida, su crítica al colonialismo no viene motivada por la creencia en un ámbito - moral o cultural - de "exterioridad" frente a occidente, y mucho menos por la idea de un retorno nostálgico a formas tradicionales o pre-

$2 \quad$ Es el caso de los programas de Antropología de las universidades Javeriana y del Rosario. capitalistas de existencia. Ellos saben perfectamente que la occidentalización es un fenómeno planetario sin retorno y que el único camino viable para todo el mundo es aprender a negociar con ella. En este sentido, como lo afirmara Spivak, su actitud frente a la globalización es la de una "crítica permanente frente aquello que no se puede dejar de desear" (Spivak 1996: 27-28). Y sus metodologías preferidas son la "reconstelación" y la "catachresis", esto es, el uso estratégico de las categorías más autocríticas desarrolladas por el pensamiento occidental para recontextualizarlas y devolverlas en contra de sí mismo (Castro-Gómez \& Mendieta, 1998).

En este contexto, la discusión sobre el estatuto epistemológico de las Ciencias Sociales, ha convocado la necesidad de reafirmar procesos de interdisciplinariedad, necesarios en la manera de analizar los procesos sociales. Realmente, muchos de estos procesos han tratado de rearmar un rompecabezas de estudios sobre las intervenciones del hombre en el mundo que a raíz de la hiperdivisión de campos del conocimiento, generó una crisis del sentido de la información y conocimiento obtenidos en cada uno de ellos, particularmente en las Ciencias Sociales. La Antropología por su enfoque ha tenido la particularidad de poner un pie en diferentes campos disciplinares tanto en las Ciencias Sociales como en las denominadas ciencias exactas. Esto ha permitido establecer una permanente crítica a su estatuto epistemológico.

Para el caso que interesa, se plantea como apoyo el trabajo y la genealogía desarrollada en la relación Antropología - Educación. De este campo de la Antropología es importante resaltar el acercamiento realizado hacia la Psicología, que dio diversos frutos en la percepción del proceso educativo; allí se constituyó la escuela de cultura y personalidad, representada en principio por Margaret Mead, (1972) y Ruth Benedic, (1967) y, posteriormente, por Kardiner, (1972) y Wallace (1972). En los últimos años, esta corriente ha centrado sus estudios en la investigación de fenómenos de transmisión -adquisición de cultura, entendiendo la educación como un proceso de transmisión de cultura. Entre los investigadores que han fortalecido dicho esquema se encuentran Gearing (1984), Splinder (1984) y 
Kimball (1974), García Castaño y Velasco (1994). Desde la década pasada se ha profundizado el esquema para otorgarle más cobertura hacia diversos fenómenos contemporáneos como los ambientes digitales y su relación con la educación. De sus orientaciones y representantes se hablará en el siguiente apartado.

\section{Etnografía aplicada en ambientes digitales}

La nueva geografía es virtual y el centro de resistencia política y cultural tiene que afirmarse en este espacio electrónico.

Un nuevo espacio de transformaciones socioculturales de enorme velocidad de realización, sustentada en nuevos códigos y lenguajes, se encuentra abierto para la investigación etnográfica; afecta todos los campos de la actividad humana en los entornos sociales e individuales y las relaciones sociales.

Dicho espacio no es el espacio físico, nos referimos a aquellas regiones y comunidades virtuales, pues las "nuevas tecnologías" han terminado de abolir el concepto tradicional de distancia física y geográfica:

\begin{abstract}
¿Qué menos puede hacer una etnografía que ya no tiene siquiera "calles" por donde transitar ni "cuerpos" a los que observar? [...] En lugar de estudiar lugares -físicos- y emplazamientos, esta etnografía [...] ha de abocarse a investigar trayectorias, vínculos y líneas de flujo. [...] el espacio concreto en que se mueve una etnografía del ciberespacio no es acotable ni similar a los clásicos emplazamientos físicos que han ocupado los grupos sociales que han acostumbrado a estudiar los antropólogos (Mayans I Planells, 2003b).
\end{abstract}

En efecto, las Nuevas Tecnologías de la Información y la Comunicación (NTIC) y en especial la World Wide Web, se constituyen en instrumentos o técnicas de trabajo para el investigador, tanto en el proceso etnográfico: recogida de datos, organización, análisis e interpretación de la información, como en la redacción y producción del producto o texto etnográfico: la presentación y difusión de los resultados. Igualmente, permite analizar la interacción de los individuos en el ciberespacio, a partir de la creación de una denominada cibercultura en la Red, entre otros aspectos.

Si decimos que las nuevas tecnologías y las comunicaciones mediadas por el ordenador están produciendo importantes cambios en nuestras sociedades, no es sólo producto de la tecnología, sino del uso que se está haciendo de ella, porque en definitiva, el ciberespacio es un lugar en el que se actúa, es un espacio social producto del flujo de información creado entre diferentes usuarios (Moraes, 2005).

Frente a esta realidad, los métodos tradicionales de investigación, se encuentran ante la necesidad de estar abiertos a nuevas propuestas y seguramente, de adaptarse metodológicamente a los nuevos paradigmas, ya que es sabido por todos que cualquier pretensión exclusivista de una parcela de la realidad social desde una sola disciplina es parcial, errónea y conduce a confusiones. En otras palabras, es una invitación a "la innovación experimental y a nuevas reorientaciones de investigación que van más allá de lo hasta ahora establecido desde los modelos clásicos de la etnografía y su modelo académico tradicional" (Téllez, A., 2002a). Es, como afirma Mayans I Planells, (2003a) "tener una sensibilidad metodológica especial a la hora de tratar la forma material de las socialidades ciberespaciales".

Las Ciencias Sociales y el investigador no se pueden desentender del estudio de las implicaciones socioculturales de la importante innovación tecnológica que representa la Red. Por una parte, a dichas ciencias, les corresponde observar todos estos procesos, identificar cuánto haya en ellos de nuevo, viejo y novedoso. Hacen falta estudios empíricos y nuevos modelos teóricos para analizar, describir e interpretar estas nuevas realidades emergentes. Por otra, el mero hecho de observar algo que está transformando nuestro entorno más inmediato a una gran velocidad y sin saber hasta dónde puede llegar, es una tarea que difícilmente al investigador puede pasarle inadvertida. Este hecho, puede arrastrar al investigador hacia aguas más tranquilas pero a la vez más alejadas de la realidad 
social y cultural del momento, y se produce cuando, observando estos grandes cambios que ya se están produciendo y delante de esta vertiginosa velocidad en que van sucediéndose los acontecimientos, el investigador pasa a tomar un papel activo, de manera militante o no, en el lado de los que se sienten amenazados como integrantes de una cultura que puede sufrir importantes transformaciones y en un lapsus de tiempo podríamos decir que insignificante.

Es así como la etnografía constituye un "viejo" método de trabajo e investigación perfectamente válido para aproximarnos a las "nuevas" tecnologías, una vez "adaptado" a las peculiaridades concretas con las que "lo ciborg" nos desafía Mayans I Planells (2003a).

El debate ahora se centra en la cuestión de si el clásico método etnográfico es válido para el estudio de las relaciones sociales y la cultura a través de Internet; en otras palabras, si las metodologías que ofrece la etnografía tradicional, esto es, las utilizadas en nuestro mundo real, son susceptibles de ser implementadas en el mundo virtual.

La etnografía virtual comprende plenamente la metodología etnográfica clásica en todos sus presupuestos ya que, al fin y al cabo, los cambios en el género (que no en la sustancia) del objeto de estudio, no derivan necesariamente en alteraciones esenciales de la praxis empírica. Es decir, la etnografía como método inductivo, practicado, holístico, transcultural y que tiene por finalidad describir y analizar el sustrato cultural de las construcciones sociales permanece inalterado cuando la investigación se traslada al ciberespacio (Domínguez, 2006).

En el debate existente sobre el estudio de la tecnología, Internet, el ciberespacio y la etnografía virtual, encontramos a varios teóricos, quienes, unos son optimistas y entusiastas ante los efectos sociales del uso de estas tecnologías, otros, en cambio, más pesi- mistas, pues denuncian los efectos sociales peligrosos de la red ${ }^{3}$; sin embargo:
A estas alturas, Internet no es ninguna novedad. Ya no hay que descubrirlo ni empezar cualquier artícu- lo sobre 'lo social' en Internet explicando primero qué es ni cómo surgió. Por fin, va acabándose la etapa del deslumbramiento y los desmesurados alegatos de apologetas y apocalípticos se van fun- diendo en un discurso menos exclamativo y más realista. [...] Internet como instrumento ha pasado a ser algo cotidiano que hemos integrado, sin traumas aparentes, en nuestras prácticas (Mayans I Planells, 2002b).

En los primeros estudios realizados sobre el tema se afirmaba que este nuevo medio empobrecía la comunicación (Hine, 2004). Así el desarrollo de Internet y las denominadas comunidades virtuales representarían un peligro para el desarrollo de vínculos sociales "reales".

Otros teóricos comenzaron a señalar las interesantes formas de interacción que se producían en el ciberespacio. Rheingold (1996) fue el primero en 1993 en hablar de las comunidades virtuales como espacios que surgen a partir de intereses compartidos. Existirían así lugares de socialización en el ciberespacio como agregaciones culturales que surgen cuando un número suficiente de gente se encuentra con otros el suficiente tiempo en el ciberespacio.

A partir de aquella primera definición de comunidades virtuales, se pasó a considerar al ciberespacio como un lugar donde se producían relaciones sociales significativas y, por tanto, donde se "producía cultura", se lo empezó a considerar así como un contexto cultural.

Existen numerosos críticos en relación con si a tales formaciones se las puede llamar comunidad e inclusive si se puede hablar de virtualidad (García, 2002). Hay quienes ven a Internet sólo como un medio para

\footnotetext{
3 No queremos entrar aquí en la discusión sobre si podemos hablar de la existencia de comunidades virtuales o no, sólo indicar que el debate sobre este tema aún no está cerrado. Ver el excelente estudio de Graham (2001, pp. 17-30).
} 
acortar distancias, sólo como nuevas mediaciones, como un nuevo medio de comunicación y no como una nueva realidad. Así mientras algunos hablan de la existencia de "formaciones sociales no-tradicionales" (Jones, 2003), otros se limitan a hablar de la existencia de "pseudo-comunidades" (Hine, 2004).

Quizás debamos considerar la idea de comunidad virtual como metáfora, como narrativa, como "comunidad imaginada"(Anderson, 1993), Christine Hine expresa que:

al considerar el ciberespacio como nuevo contexto se han privilegiado los análisis de lo que ocurre $O n$ line sin relacionarlo con lo que sucede Off line. Desde su punto de vista hay que dar cuenta de Internet en sus dos dimensiones: como cultura y como artefacto cultural (Hine, 2004).

Son estos dos aspectos los que lo convierten en un recurso para el trabajo de campo etnográfico.

Junto al excelente estudio de Christine Hine (2004), existen otros recientes, compendiados en las obras de: Daniel Miller y Don Slater (2000), David Bell (2001), Frank Schaap (2002), Joan Mayanas (2002c), Mia Consalvo y Sussana Paasonen, (2002), Nicole Constable, (2003), William J. Mitchell (2003), Elizabeth Buchanan (2004), y Jeff Rice (2004).

Es un hecho que una nueva

revolución tecnológica masiva, Internet y las comunicaciones mediadas por ordenador, han alterado en menos de un decenio las costumbres comunicativas, expresivas, lúdicas, laborales, económicas, políticas y culturales de un gran segmento de la población (occidental) (Mayans I Planells, (2003b). [...] Como investigadores sociales, en estos años de descubrimiento y acostumbramiento, la línea que parece trazarse marca un camino desde lo que fueron, inicialmente, estudios sobre aquello que de "social" identíficábamos en o alrededor de las -nuevas- tecnologías de la información y la comunicación hacia lo que deberían ser, cada vez más claramente, investigaciones sobre la sociedad digital (Mayans \& Planells, 2003a).
Por otro lado, los trabajos de Lave \& Wenger (2003), aportan elementos metodológicos valiosos en los análisis de comunidades educativas; el análisis situacional centrado en el sentido del aprendizaje dentro de las comunidades de práctica. A estas ideas las llaman proceso de participación periférica legítima, puesto que el nuevo participante, que se mueve de la periferia de la comunidad hacia el centro, llegará a ser más activo y a estar más comprometido con la cultura y, por ello, asumirá una nueva identidad.

Esta técnica será extremadamente valiosa para observar el grado de articulación que realizan los miembros de las comunidades educativas analizadas en torno al uso y aplicación de ambientes digitales en procesos de educación superior.

Muchos de estos elementos etnográfico-metodológicos han venido siendo enfocados dentro de los ambientes digitales; allí, igualmente, existen varias perspectivas teóricas que siguiendo el ejercicio de integración que realiza el grupo de etnovirtual (Coordinado por: Adolfo Estalella, Elisenda Ardevol Piera, Daniel Domínguez, Édgar Gómez Cruz), enmarcamos dentro de lo que se llamaría "Etnografías de lo digital".

Por "etnografías de lo digital" queremos designar las formas de hacer etnográfico en el espacio de interacción que configura Internet y las tecnologías digitales (teléfonos móviles, fotografía digital, redes inalámbricas, etc.). De manera que en el concepto de "etnografías de lo digital" aglutinamos la etnografía virtual (Hine, 2000), la etnografía del ciberespacio (Hakken, 1999), la etnografía de/en/a través de Internet (Baulieau, 2004), la ciber-etnografía (Escobar, 1994), etc. (Domínguez et al. 2006).

Lo anterior se debe a que se pretende, principalmente, abordar el problema de las representaciones que se alojan dentro de los espacios que rodean la relación entre discurso y práctica pedagógica en relación con la ecuación aplicada en ambientes digitales. De allí que el enfoque metodológico se centre en identificar las relaciones con lo digital, esto no obstante, de 
la observación y la indagación que pasa desde los lugares estrictamente digitales, deambula de acuerdo con el mundo de conexiones de las poblaciones seleccionadas, por lugares presenciales y la manera como se interconectan lugares, discursos y prácticas de lo digital con lo presencial.

\section{Enfoque etnográfico de las dos investigaciones}

De acuerdo con las consideraciones anteriores de orden teórico y práctico ${ }^{4}$, la investigación de campo de las dos investigaciones propuestas, se circunscribe dentro de las aproximaciones metodológicas a los ambientes virtuales; esto es, realizar nuestra práctica etnográfica en este contexto. Con ello, queremos compartir una experiencia de investigación de dichos ambientes como objeto, "campo" y recurso etnográfico.

En razón a los antecedentes planteados, el trabajo etnográfico de las dos investigaciones mencionadas, se basa en la observación participante, con un alto contenido de interpretación de los diferentes discursos de lo observado, para ello la antropología simbólica ofrece varias herramientas metodológicas y experiencias de investigación social aplicadas en ambientes virtuales (Turkle, 1995, Mayans I Planells, 2001), adicionalmente se complementa con dicha perspectiva la visión de Jesús Ibáñez (1979) sobre los grupos de discusión ${ }^{5}$. Lo anterior, en seguimiento al análisis del

4 La presente investigación de campo no desconoce, los trabajos que se vienen adelantando sobre la investigación etnográfica en ambientes virtuales: 1. Grupos de investigación y de discusión, por ejemplo, "Cultura libre y retos a la investigación en/ de Internet y las tecnologías de la información" coordinador por Yann Bona Beauvols y Juan Carlos Aceros G.; el grupo Etnografía virtual: grupo de trabajo en el OCS (Observatorio para la CiberSociedad), coordinado por Joans Mayans I Planells. 2. Para el caso de los grupos de discusión, por ejemplo: QUALRS-L Qualitative Research for Human Sciences; Aom-Resch, Qualitative Research, Qual-software, Qsr-forum, ATLAS-ti, 3. Las listas de correo. Para una mayor información sobre los grupos de discusión y las listas de correo, remitimos al trabajo de Pablo Gustavo Rodríguez: "Grupos de discusión sobre metodología de investigación cualitativa asistida por computadora en Ciencias Sociales".

5 Una de las principales aportaciones de Jesús Ibáñez a la sociología fue su fundamentación del "Grupo de discusión" como herramienta de investigación sociológica. Para Ibáñez "[...] si las técnicas cuantitativas investigan el sentido producido, los hechos, la técni- discurso de la población analizada, en dos sentidos, el de la interpretación del investigador basado en su observación participante y el de la interpretación del investigado basado en la publicación de su discurso, de su manera de enunciar las temáticas planteadas en el problema de investigación.

El esquema metodológico, sigue el mismo enfoque del marco conceptual sobre el cual se analizan las relaciones entre discurso y práctica pedagógica en las comunidades educativas seleccionadas para el estudio como se menciona más adelante. Para el caso de ambas investigaciones, una se plantea desde lo antropológico, y la otra, desde lo semiótico, ambas con el mismo enfoque metodológico etnográfico. Veamos por separado la explicación de cada una:

\begin{tabular}{|c|c|}
\hline Investigación 1 & $\begin{array}{l}\text { Educación virtual: del discurso a las } \\
\text { prácticas pedagógicas. Un estudio } \\
\text { etnográfico en seis instituciones de } \\
\text { Educación Superior en Colombia. }\end{array}$ \\
\hline Objetivo & $\begin{array}{c}\text { Realizar un estudio etnográfico que } \\
\text { identifique relaciones entre el discurso y } \\
\text { las prácticas pedagógicas de la educación } \\
\text { virtual en seis Instituciones de Educación } \\
\text { Superior en Colombia. }\end{array}$ \\
\hline Investigador & Julio Ernesto Rojas Mesa \\
\hline Investigación 2 & $\begin{array}{c}\text { Fundamentación teórica de la educación } \\
\text { virtual en la educación superior en } \\
\text { Colombia. }\end{array}$ \\
\hline Investigador & Carlos Bernal Granados \\
\hline Objetivo & $\begin{array}{l}\text { Presentar una fundamentación teórica de } \\
\text { la educación virtual que permita identificar } \\
\text { los desarrollos de la educación tradicional } \\
\text { en la implementación de tecnologías en } \\
\text { los procesos pedagógicos de la educación } \\
\text { superior en Colombia. }\end{array}$ \\
\hline
\end{tabular}

ca del grupo de discusión investiga el proceso de producción de sentido, que no es más que la reproducción de la unidad social de sentido". El lenguaje permite interpretar el mundo cognitivo en su dimensión estructural del componente simbólico. El grupo de discusión es una muestra estructural con características propias. El grupo ha de ser considerado como un todo en el que cada integrante al participar, va generando cambios tanto en su discurso propio como en el grupal, su importancia radica en concebir la discusión como la unidad mínima de interacción social (Pask) que a partir de este juego reproduce el orden social. Recuperado el 3 de abril de 2008 de: http: //teoriaypraxissociologica.wordpress. com/2007/12/20/ibanez-y-el-grupo-de-discusion/ 


\section{Investigación 1: enfoque antropológico}

Para la investigación titulada Educación virtual: del discurso a la práctica pedagógica. Estudio etnográfico en seis instituciones de educación superior en Colombia, se trabaja desde una perspectiva antropológica que entiende la virtualidad como un proceso connatural al ser humano y su proceso de hominización, que por tanto, no es equivalente al espacio digital exclusivamente. Define las instituciones educativas como sistemas culturales en micro dentro de la sociedad colombiana y en el mundo de manera global (es decir, sistemas vistos como totalidades en interacción con otros sistemas en espacios y procesos similares).

Teóricamente la perspectiva presentada se basa en un triangulo conceptual constituido por el mito, el rito y el territorio como dispositivos de agenciamiento de identidades, relaciones de pluralidad, alteridad e interacción que determinan la construcción de interfa- ces, el uso de artefactos y la creación de ambientes y comunidades en permanente transito de paradigmas y sistemas simbólicos (Auge, 1995).

Las instituciones educativas se entienden como sistemas culturales, constituidos como territorios o escenarios de identidades múltiples que interactuaran dentro de una noción de alteridad construida sobre los mitos y ritos de la institución educativa, generando una representación de pluralidad, de grupos, de pensamiento y de producción de sentidos. Como sistema, hay una íntima interrelación entre cada nodo, de tal manera que no hay uno primero que otro, o no hay uno más relevante que otro, cada uno establece allí un acto de transmisión, de reproducción o de producción que transforma permanentemente el sistema.

El siguiente esquema gráfico, describe la manera como se entiende dicha perspectiva antropológica del trabajo de campo.

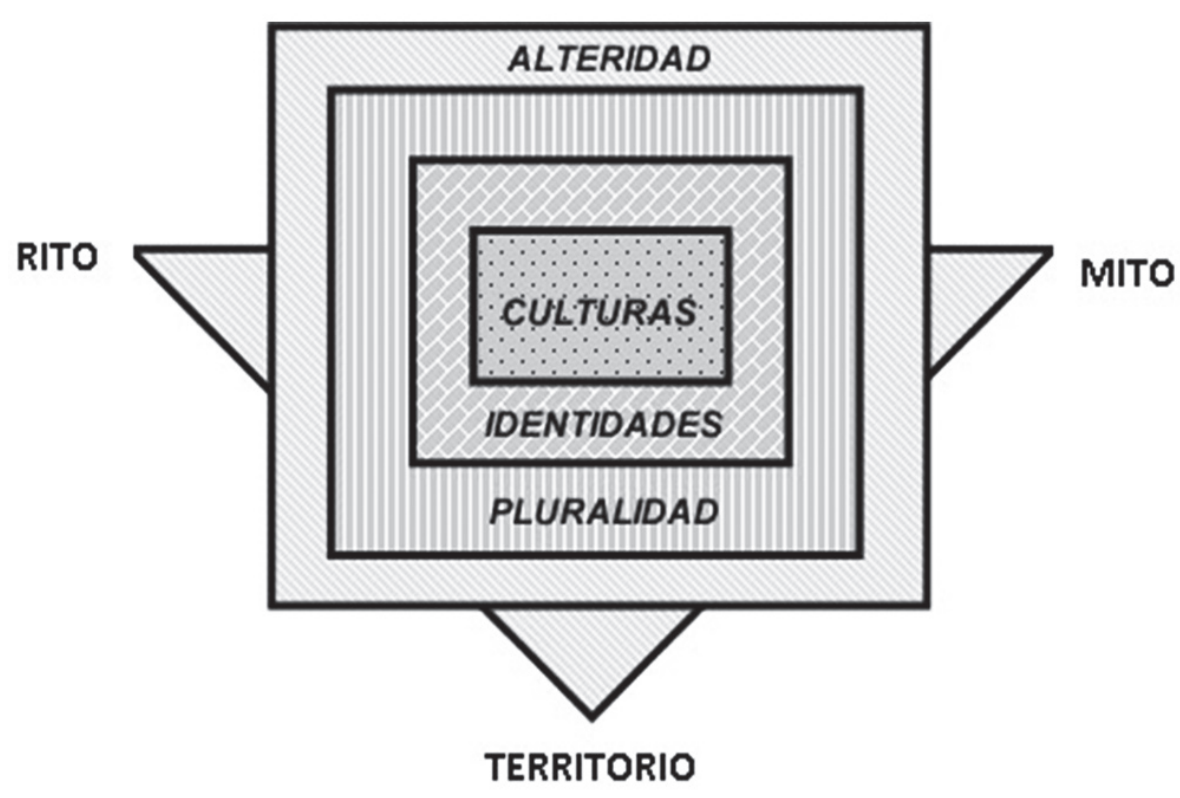

Gráfico 1. Perspectiva antropológica del trabajo de campo

De allí, se desprenden las temáticas planteadas en las encuestas y las entrevistas, así como en las guías de orientación de la observación etnográfica y el diario de campo elaborado durante el trabajo de investigación.
Investigación 2: enfoque semiótico

Para la investigación titulada: Fundamentación teórica de la educación virtual en la educación superior en Colombia, se trabaja desde una perspectiva semiótica. 
En particular de la semiótica virtual, esto es, de las nuevas relaciones que se han originado entre semiótica e informática y entre lenguaje e Internet.

Etimológicamente, la palabra semiótica deriva de la raíz griega semeîon (signo) y sema (señal); en consecuencia, la semiótica se ocupa del estudio de los signos (o de los lenguajes naturales o artificiales en cuanto sistema de signos) y su significado, naturaleza, clases, tipos y en especial, mediante la articulación de tres funciones esenciales a su objeto, como: instaurador de sentido, facilitador de relaciones comunicativas $y$, configurador de cultura.

Desde esta perspectiva, le compete proporcionar los fundamentos de cualquier ciencia particular que trate de signos, como la lógica, la lingüística, la matemática y recientemente la informática (Internet, ambientes virtuales), entre otras.

[...] por más que se afirme que la semiótica es una ciencia, nada más alejado de la realidad. Esta preocupación etimológica además de tratar de aclarar el proceso diacrónico sufrido por el término, abre espacio para discutir las dos grandes corrientes del siglo XX en el campo del estudio de los signos; la semiología y la semiótica. Semiología es una deformación moderna y, a veces, se utiliza semeiología, sematologia, semeiotica, tanto como "semiotics" que en inglés le da a la palabra una forma plural y sirve para denominar como ya dijimos una "ciencia". Aunque en el primer congreso de la Asociación Internacional de Semiótica se adopto la palabra "semiótica" como el término para abarcar el estudio de las tradiciones de la semiología y la semiótica general. (Del Villar, D. s.f., p. 1).

El dominio de la semiótica es amplio y diverso, el cual se manifiesta tanto en los modelos de análisis como en los campos de aplicación de la cultura en general. Otro tanto le compete a la llamada semiótica virtual de acuerdo con la propuesta de Noboa, M. (2004), Arroyo, S. (2004), Pellerey, R. (2004), Martí, D. (2004, 2007), Magariños, J. (2004), Rivas, M (2001), entre otros.
Todo lo dicho hasta ahora, explica por qué el estudio del signo se constituye en el eje de los procesos semiósicos ${ }^{6}$, ya que a partir de él se estructuran los diferentes mensajes que funcionan como cadenas de totalidad significativa, contextualizados, por una parte, en el ámbito de la comunicación verbal y no verbal, y por otra, otorgando pleno sentido, construidos desde el plano de la expresión y del contenido (Hjemslev, 1971). Nos referimos en primer lugar a los mensajes de los textos, los cuales permiten configurar el discurso; en segundo lugar, a los signos lingüísticos, icónicos, fonéticos, gustativos, expresivos, estéticos, etc.

La rápida digresión que hemos llevado a cabo, nos permite tratar brevemente dichos mensajes de la comunicación verbal y no verbal para ser estudiados y analizados desde la semiótica virtual.

En los antecedentes del presente artículo planteábamos que el nuevo espacio de transformaciones socioculturales (Internet), afecta todos los campos de la actividad humana en los entornos sociales e individuales y de relaciones sociales: Igualmente, los discursos sociales que en su proceso de transformación han originado nuevos géneros (códigos y lenguajes) que conllevan "diferentes estrategias de intercambio de información, producción, comprensión y lectura de textos" (López, 2003; García, 2005). Dichas transformaciones:

[...] se refieren en primer lugar al formato discursivo; éste tiene sus características propias que se deben a la tecnología computacional. El nuevo tipo se puede denominar el discurso virtual (por el espacio donde se elabora) o electrónico (por el medio tecnológico que lo propicia); lo mismo se refiere al concepto de escritura. Lógicamente si se trata de la noción de signo en el hábitat computacional, se le designaría de manera semejante: el signo virtual o electrónico. (Sorókina, 2004, p. 2).

$6 \quad$ La semiosis es un proceso y el análisis semiótico es el estudio del funcionamiento de esas semiosis, ya sea a posteriori, en una obra acabada, ya sea en el acto mismo de creación de la obra, desde el interior mismo del proceso semiótico. El propio análisis es proceso semiótico (Deladalle, 1996, p. 101). 
Visto lo anterior desde la investigación etnográfica virtual, y por ser un nuevo espacio abierto al investigador, falta mucho por hacer y debatir (Crystal, 2002; Lavid, 2005; Rodríguez, 1999, 2007; García, 2005).

El soporte básico en Internet es el texto, el hipertexto y los modelos hipermedia, como la vía principal de comunicación entre los usuarios de la Red, lo cual articula y fortalece la perspectiva del relativismo cultural que analiza la cultura como un texto. Pero la referencia no está dirigida a un texto escrito y "muerto" sino a un texto ya no individual sino colectivo, en continuo proceso de redacción, de remodelación, de autocorrección por parte de sus propios protagonistas. En resumidas cuentas, éste soporte básico se constituye en las "áreas donde los lenguajes informáticos y las lenguas naturales se entrelazan dando lugar a nuevas formas de comunicación, no sólo entre el hombre y la máquina, sino también entre el hombre y el hombre" (López, 2003, p. 110).

Frente a esta observación, "nos encontramos ante un fenómeno lingüístico y social de considerable magnitud", que según Joan Mayans.

La antropología lingüística, en su vertiente más pragmatista, ofrece instrumentos metodológicos de utilidad para comprender, analizar e interpretar el incontenible e inabarcable flujo textual que emanan las CMO (Comunicaciones mediadas por ordenador). (Mayans I Planells, 2003b).

Igualmente, Tatiana Sorókina, (s. f.) expresa:

El hipertexto tiene ciertas características generales que lo convierten en un fenómeno en sui generis. Desde el punto de vista de cuantía, presenta enormes cantidades de información con todas las modalidades sígnicas. Desde el enfoque organizacional, no se construye linealmente, tampoco a partir de los centros hacia la periferia, a la manera del discurso científico. Tercero, su estructura es "multiestabilizada" (Klinkenberg) por las relaciones exigidas no tanto por la lógica (una propiedad bastante restringida por el verbo escrito) como por las asociaciones miméticas (el nivel consciente $o$ subliminal). Las relaciones sígnicas forman una especie de red multidimensional (algo semejante a las redes de pesca existentes unidas entre sí) con una diferencia: la red virtual permite la integración de todo elemento nuevo en cualquiera de sus partes sin que el sistema hipertextual se rompa, sino que sólo aumenta (equivalente a mejorar) su potencial informativo. Finalmente desde la perspectiva operacional (la creación Vs la interpretación), el hipertexto se caracteriza por un cambio esencial de los papeles entre el autor (como un agente activo) y el lector (como un agente pasivo) en lo que se refiere a la producción discursiva. [... ] Desde el punto de vista de la teoría de signo, el carácter innovador de la escritura electrónica se descubre en la heterogeneidad semiótica, en primer lugar con todos los acontecimientos empírico-reflexivos. El cerebro computacional, en cierto sentido semejante al cerebro humano, contiene y "opera" con datos de distinta índole; en la pantalla del monitor se plasma la información visual, lingüística y auditiva. No es nada desconocido en los demás medios, sin embargo, existe algo que hace la pantalla 0 , más preciso, la página virtual, diferente a la página del libro (Sorókina, 2004, p. 3).

De otra parte, los iconos, se constituyen en los símbolos más utilizados en Internet. La iconografía es uno de los factores más determinantes dentro del ciberespacio, y esta iconografía ayuda a unificar a la llamada cibercultura, ya que los usuarios de la Red, conocen perfectamente el significado de cada uno de estos símbolos.

Junto al texto y a la simbología, en Internet encontramos la imagen en sucesión, el vídeo como un nuevo "texto simbólico" que debemos descifrar y analizar antropológicamente. Este soporte, el audiovisual en la Web, es más rápido que el texto y nos exige a los investigadores socializarnos en esta cibercultura para poder entender y descodificar las imágenes y las representaciones ideológicas que las sustentan. Es por ello que el investigador debe detenerse en el análisis del discurso en la Web, tanto del texto como de la imagen (estática o en movimiento).

A su vez, el investigador debe analizar cómo se construye el discurso científico y cómo se traduce en 
la cotidianidad de los sujetos cibernautas, los usos sociales de las nuevas tecnologías en diversos contextos culturales.

Por lo anterior, todos estos elementos que nos ofrece Internet se constituyen en un producto cultural, en una manifestación artística si se quiere, cargada de representaciones ideológicas llenas de información interpretable. En otras palabras, tanto el texto como la imagen en la Web (estática o en movimiento) son fuente directa de información sobre los contenidos culturales que podemos analizar en la etnografía de las instituciones educativas estudiadas.

En cuanto a las representaciones ideológicas con un elevado caudal de información interpretable, el investigador debe conocer los valores y las representaciones ideológicas principales del entorno ciberespacial en el que investiga. Así necesitará saber cuáles son los "valores centrales" dentro del ciberespacio, entre los que algunos estudiosos destacan como claves el individualismo, la iniciativa y la igualdad de oportunidades (Faura, 2000). Valores y comportamientos compartidos por muchos cibernautas a quienes se les considera que han desarrollado una "nueva cultura" en Internet (Téllez, 2002; Maldonado, 2007).

Así pues, para realizar estudios etnográficos en el soporte tecnológico de Internet se hace preciso considerar tanto las imágenes como el texto como elementos centrales para el análisis y la deconstrucción de los discursos, puesto que es necesario estudiar el formato de los materiales o datos etnográficos que se utilizan en y para la comunicación en este denominado ciberespacio.

\section{Población objetivo del estudio}

La población seleccionada para realizar el trabajo de campo de ambas investigaciones, es básicamente tres tipos; los cuales son integrantes de lo que conocemos como comunidad educativa de las Instituciones de Educación Superior en Colombia: estudiantes, docentes y académicos-administrativos (coordinadores o directores de unidades académicas, decanos, vicerrectores y rectores). A esta población se aplicarán las técnicas de recolección de información.

\section{Investigación 1}

La muestra institucional escogida fueron seis universidades de carácter, todas ellas con sede en Bogotá, planteando algunos criterios preliminares para su organización y análisis de relaciones posterior, así:

- Tres de ellas son públicas y tres privadas.

- Tres tienen modalidad a distancia o bimodal y tres tienen modalidad presencial.

- Tres tienen presencia nacional y tres tienen presencial local.

Tabla 1. Investigación 1

\begin{tabular}{|c|c|c|c|}
\hline Universidad & Carácter & Modalidad & Cobertura \\
\hline Universidad Nacional de Colombia & Pública & Presencial & Informática educativa \\
\hline Universidad Nacional Abierta y a Distancia & Pública & A distancia & Educación virtual \\
\hline Universidad Javeriana & Privada & Presencial con EAD & Informática educativa \\
\hline Universidad Santo Tomás & Privada & Presencial con EAD & Informática educativa \\
\hline Universidad Minuto de Dios & Privada & Presencial con unidad virtual & Informática educativa \\
\hline Universidad Pedagógica Nacional & Pública & $\begin{array}{c}\text { Presencial con apoyos } \\
\text { informáticos }\end{array}$ & Informática educativa \\
\hline
\end{tabular}




\section{Investigación 2}

La muestra institucional escogida fueron seis universidades. Los criterios básicos para tener en cuenta para su organización y análisis de relaciones posterior, así:
- Dos tienen modalidad a distancia o bimodal y cuatro tienen modalidad presencial.

- Una tiene presencia nacional; otra regional y cuatro tienen presencial local.

- Tres de ellas son públicas y tres privadas.

Tabla 2. Investigación 2

\begin{tabular}{|c|c|c|c|}
\hline Universidad & Carácter & Modalidad & Cobertura \\
\hline Universidad de la Salle & Privada & Presencial & Informática educativa \\
\hline Universidad Tecnológica de Pereira & Privada & Presencial & Informática educativa \\
\hline Universidad Industrial de Santander & Pública & Presencial con EAD & Informática educativa \\
\hline Universidad del Valle & Pública & Presencial & Informática educativa \\
\hline Universidad de los Andes & Privada & Presencial & Informática educativa \\
\hline Universidad Santo Tomás & Privada & Presencial con EAD & Informática educativa \\
\hline
\end{tabular}

\section{Técnicas de recolección de información}

\section{Generalidades}

En lo relacionado con las técnicas de recolección de información utilizadas de manera tradicional por la etnografía, se han realizado algunos acercamientos sobre su uso en ambientes digitales y en un alto porcentaje de sus casos son altamente adecuadas para adelantar este tipo de investigación, tal cual como se mencionó en los antecedentes. En otros casos, la etnografía a través de sus estudios ha actualizado otras técnicas y las ha incorporado en su corpus metodológico para el abordaje de análisis de ambientes digitales.

A continuación relacionamos las técnicas de recolección de información por utilizar en ambas investigaciones y su relación con el ambiente digital.

A continuación el esquema de técnicas de recolección de información tradicionales combinadas con los nuevos recursos digitales:

Tabla 3. Técnicas de recolección de información utilizadas

\begin{tabular}{|c|c|c|}
\hline Técnicas tradicionales & $\begin{array}{c}\text { Técnicas adaptadas o actualizadas en } \\
\text { sistemas digitales }\end{array}$ & $\begin{array}{c}\text { Técnicas surgidas en el uso de los } \\
\text { sistemas digitales }\end{array}$ \\
\hline Observación participante & Observación participante On line & Utilizada como navegación en Web \\
\hline Diario de campo & $\begin{array}{c}\text { Se adapta el diario de campo al formato } \\
\text { digital y la escritura on line }\end{array}$ & Utilizado como bitácora \\
\hline $\begin{array}{c}\text { Conversatorios o paneles de } \\
\text { invitados }\end{array}$ & \begin{tabular}{c} 
Utilizado como Audio conferencia discusión y foros \\
\hline $\begin{array}{c}\text { Entrevistas colectivas } \\
\text { Entrevistas individuales }\end{array}$
\end{tabular} & Audio conferencia \\
\hline Encuestas & $\begin{array}{r}\text { Es tradicional y solamente se actualiza en } \\
\text { el sistema digital }\end{array}$ & Utilizado como Chat \\
\hline
\end{tabular}




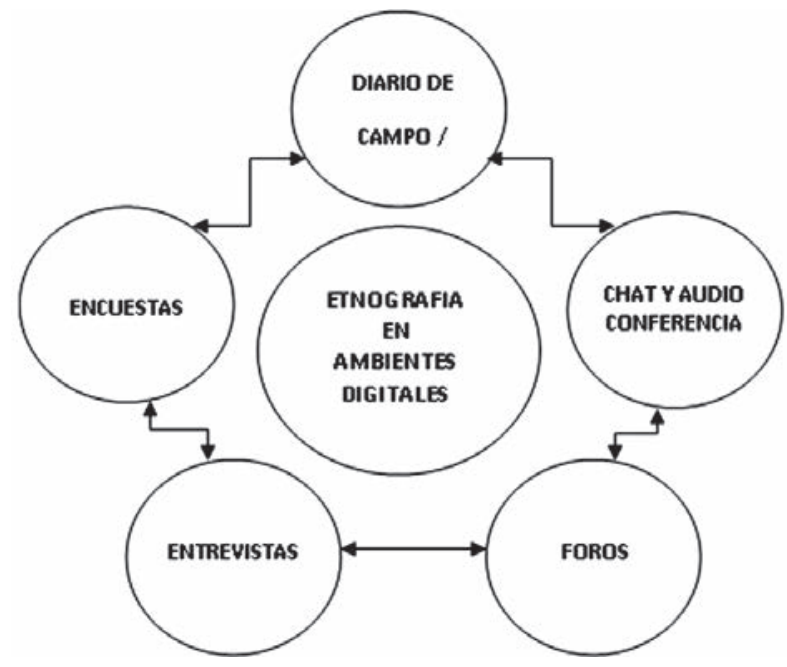

Gráfico 1. Técnicas de recolección de información utilizadas

Las muestras, a las que se aplicarán las encuestas serán aleatorias y con carácter abierto a la participación del total de la población de cada grupo escogido. Sobre el total de aquéllos que respondan las encuestas se realizarán los análisis de relaciones estadísticas.

\section{Las encuestas}

Se diseñaron dos tipos: un primer tipo de formularios elaborados para las universidades, a fin de ser respondidos de manera institucional. En ellos se solicita información de tipo general relacionada con la institución y sus procesos de aplicación de ambientes digitales, tanto en lo académico como en lo administrativo.

Un segundo tipo de formularios elaborados para ser respondidos, de manera individual, por los miembros de los distintos grupos identificados como población objeto de la investigación. Es así como se elaboraron encuestas para estudiantes, docentes y académicosadministrativos.

\section{Las entrevistas}

Se realizaran bajo modalidad presencial y en ambiente digital. Igualmente se realizarán de manera individual y grupal así:

- Entrevistas individuales: realizadas a una muestra de población de cada tipo seleccionada según su posición frente a estos procesos de tal manera que se pueda revisar un espectro de diferentes perspectivas del problema de investigación. En ambiente digital esta técnica se aplicará como chat $o$ audio conferencia

- Entrevista colectiva, aplicada a manera de conversatorio, convocando a aquéllos que tengan experiencia en el tema para que profundicen un poco más sobre las categorías de análisis propuestas en la investigación. En ambientes digitales, esta técnica se utilizará bajo las figuras de audio conferencia y foro de opinión.

\section{El diario de campo o bitácora}

En él se centra mucho del registro realizado en la investigación, en este caso se llevará en línea y tendrá dos dimensiones:

- Una escritura que tradicionalmente realiza el investigador y que tiene que ver con sus percepciones $\mathrm{y}$ análisis personales acerca del desarrollo del trabajo de campo y del contexto en que éste se desarrolla. Éste será de acceso exclusivo del investigador y se consulta y edita con clave de acceso

- La otra escritura estará abierta a los usuarios, población objeto de la investigación, y se realizará como una escritura de experiencias y vivencias relacionadas con el uso o adaptación de los medios digitales en su mundo cotidiano y profesional. A este lugar se le ha denominado Bitácora y en él todos pueden consignar sus comentarios, lo que equivaldrá al lugar de lo emic o la visión del investigado.

\section{Categorías de análisis para el desarrollo del trabajo de campo}

\section{Generalidades}

De acuerdo con el marco teórico propuesto, se plantean tres dimensiones generales e interrelacionadas, de análisis de la población objeto de la investigación. 
Estas tres dimensiones son Mito/discurso; Ritual/práctica; y Territorio/escenarios.

Son dimensiones de análisis, interrelacionadas entre sí, puesto que desde la visión antropológica trabajada, la cultura es un tejido de diferentes significados construidos desde expresiones culturales del mundo cotidiano y materializado de las sociedades (significados sobre las reglas que nos llevan hasta sentidos religiosos y espirituales, guerras causadas generadas por formas particulares de vivir el odio, el amor o la nostalgia etc.) (Geertz, 1997). De allí, que para este análisis, sea fundamental mirar estos grupos con herramientas diseñadas y ordenadas de manera sistémica, interrelacionada. Se habla entonces de un triangulo de análisis compuesto por tres categorías principales: mito-rito-territorio. De ellas se desprenden dos enfoques operativos replicados en el desarrollo de cada dimensión: enfoque pedagógico-filosófico y enfoque tecnológico-comunicativo.

El mito, ofrece una ordenación propia de la realidad humana -ni superior ni inferior a la que proporciona la razón- que se debe conocer e interpretar (Cassirer, 1984; De Certau, 1999) de hecho la intención que se tiene en este estudio, y que refleja el ambiente de la última época en relación con las explicaciones que el hombre se ofrece acerca del mundo, es la búsqueda de nuevos modelos de interpretación del mito que se distancien de la tradicional dicotomía mito y logos heredada de la antigüedad clásica occidental (Duch, 1998). El reconocimiento a los contextos multiculturales y con ello, la aceptación de diferentes interpretaciones de la realidad y la naturaleza, ha contribuido a ello y es lo que permite que en este trabajo se hable con libertad de diferentes lógicas, sin que por ello se entiendan todas dentro de la categoría de lo "racional", entendido y definido de una manera univoca y propia de la ilustración occidental ${ }^{7}$ (Strauss, 1987).

7 Es importante señalar aquí el distanciamiento que este trabajo asume, acerca de las corrientes antropológicas clásicas que han demarcado el mito como un relato perteneciente a escenarios fantásticos o mágicos de la cultura, pero universaliza el mito desde una perspectiva estructuralista de la Antropología. De otras corrientes clásicas que han teorizado sobre el mito y su
El mito entonces, se manifiesta como matriz y expresión contextual e histórica, por esta razón los mitos son dinámicos y se transforman de manera constante en respuesta a las situaciones socioculturales de su tiempo y contexto (Durant, 2003; Gibson, 1991; Gadamer, 1977; Heidegger, 1994; Lorite, 1984). Esta misma razón es la que nos permite entender el mito como connatural con el rito; es decir, la práctica y con el territorio, es decir, los contextos sobre los que un determinado grupo afinca diferentes representaciones de su mundo (Díaz, 1998; Castells, 1998; Deleuzze, 1988; Serres, 1994). Se encuentra en la triada mito/discurso-rito/práctica-territorio/ contexto, una vigencia fundamental para entender las diferentes relaciones establecidas en las comunidades de educación superior estudiadas aquí, articulado de manera sistémica a nociones de historia y tiempo, relacionadas con el ritual, así como con escenarios y contextos; en algunos casos de espacialidad física, en otros de territorialidad simbólica y ciberespacial (Dery, 1998; Heim, 1993. Levy, 1999; Stone, 1993).

Cada dimensión se materializa a través de dos enfoques generales para su análisis a saber:

- Enfoque filosófico-pedagógico.

- Enfoque tecnológico-comunicativo.

Estos enfoques, reúnen una serie de variables de análisis planteadas para ser resueltas, en el caso de las encuestas del segundo tipo de formularios propuesto ${ }^{8}$, sobre el rango de tres indicadores o ítems de respuesta. Los indicadores están construidos sobre una escala Likert $^{9}$ de valoración planteada en tres momentos

interpretación como elemento en las sociedades y que en este trabajo se leen con la claridad de sus aportes en el momento histórico específico, pero también con la certeza de que otras lecturas las han desbordado y revaluado. Se puede ver: Eliade (1979; 1973; 1974; 1972); Frazer (1991); Durkheim (1968). Godelier (1974), Malinowski (1974), Boas (1948).

8 Población objeto de la investigación: estudiantes, docentes y académicos-administrativos.

9 Es un tipo de instrumento de medición o de recolección de datos que dispone el investigador social para medir las actitudes. Consiste en un conjunto de ítems bajo la forma de afirmaciones o juicios ante los cuales se solicita la reacción (favorable o desfavorable, positiva o negativa) de los individuos. 
de respuesta que van desde un lugar de valoración positiva del tema, pasando por una valoración normal, hasta una valoración negativa del tema.

La ruta para el desarrollo de las variables está definida de la siguiente manera: en la primera dimensión se recogen la mayoría de las variables y en las siguientes dimensiones se mantiene un alto porcentaje y se presentan aquéllas que es pertinente modificar o cambiar de acuerdo con la naturaleza de la dimensión.

\section{Dimensiones}

\section{Dimensión discursiva}

Enfoque filosófico-pedagógico

- Variable 1: naturaleza de los ambientes digitales.

- Variable 2: impacto del uso de ambientes digitales en procesos educativos.

- Variable 3: sentido de responsabilidad o autonomía construido en relación con el uso de ambientes digitales.

- Variable 4: sentido del trabajo realizado en ambientes digitales.

- Variable 5: apropiación de conocimiento en ambientes digitales.

- Variable 6: reconocimiento y aceptación del puesto del ser humano frente a la propia tecnología que crea y utiliza, por ejemplo, para ambientes digitales.

- Variable 7: la tecnología como humanizadora o deshumanizadora del hombre y del entorno educativo.

- Variable 8: evaluación en ambientes digitales.

- Variable 9: el material educativo (objetos de aprendizaje). Enfoque tecnológico-comunicativo.
- Variable 10: sentido de la comunicación permitida por los recursos digitales.

- Variable 11: los enlaces o links.

- Variable 12: instrumentalización de los ambientes digitales.

- Variable 13: arquitectura de los ambientes digitales.

- Variable 14: prospectiva de los ambientes digitales.

- Variable 15: la filosofía de la tecnología como espacio de la cultura humana.

\section{Dimensión práctica}

Enfoque filosófico-pedagógico:

- Variable 16: nivel de interacción con los ambientes digitales.

- Variable 17: producción y uso de herramientas digitales.

- Variable 18: la tecnología como humanizadora o deshumanizadora en la práctica pedagógica en ambientes digitales.

- Enfoque tecnológico-comunicativo.

- Variable 19: relación con los aparatos digitales.

- Variable 20: navegación en internet.

\section{Dimensión espacial}

Enfoque filosófico-pedagógico:

- Variable 21: elección y utilización de los ambientes digitales en el ciberespacio.

- Variable 22: Encuentro social y aprendizaje en el ciberespacio. 
- Variable 23: relaciones y comunicación pedagógica, social y tecnológica en el ciberespacio.

- Variable 24: control y valoración de aprendizajes de los usuarios en el ciberespacio.

- Variable 25: creación, socialización, evaluación e intercambio de materiales pedagógicos en el ciberespacio.

- Variable 26: ibicación del hombre y su proceso de aprendizaje en el ciberespacio.

Enfoque tecnológico-comunicativo.

- Variable 27: conocimiento, utilización e intercambio tecnológico (hardware y software) en el ciberespacio.

- Variable 28: comunicación bidireccional e interactiva en el ciberespacio entre los usuarios de ambientes digitales.

En síntesis, este artículo plantea la necesidad de realizar una primera reflexión acerca del estado del arte de las investigaciones realizadas por los autores y el desarrollo de la propuesta metodológica para Ilevarlas a cabo. Es importante resaltar que la materialización de esta propuesta metodológica tiene una infraestructura digital de apoyo. En una página Web, dedicada al trabajo de campo de las investigaciones, se publicaran y diligenciaran los formatos de encuestas planteados desde las variables arriba mencionadas. En ella podrán tener acceso todos los estudiantes, docentes y administrativos de las doce universidades muestra escogidas para el desarrollo del trabajo de campo. Igualmente allí se publicaran informaciones y otros recursos tecnológicos propicios para el desarrollo de las investigaciones presentadas (foros, chats, links, etc.). Para la Universidad Santo Tomás esta información será de gran ayuda en la consolidación de su proyecto de articulación de tecnologías en los procesos académicos.

\section{Referencias}

Abril, G. (Junio de 1998). Sujetos, interfaces, textura. Revista de Occidente, 206, 59-76.

Anderson, B. (1993). Comunidades imaginadas: reflexiones sobre el origen y la difusión del nacionalismo. México: Fondo de Cultura Económica.

Arroyo, S., et al. (Abril-mayo de 2004). Vinculación de la semiótica y la informática: indexación digital de telediarios. Razón y Palabra. Primera revista electrónica en América Latina especializada en comunicación, 38. Recuperado el 3 de mayo de 2008 de: http: //www.razonypalabra.org.mx/ anteriores/n38/.

Atkinson, P. \& Hammersley, M. (2001). Etnografía. Métodos de investigación. Barcelona: Paidós.

Auge, M. (1995). Hacia una Antropología de Ios mundos contemporáneos. Madrid: Gedisa.

Baulieau, A. (2004). La etnografía de/en/a través de Internet. (hace parte de una cita textual del grupo etnovirtual)

Bell, D. (2001). An Introduction to Cybercultures. Londres: Routledge.

Benedic, R. (1967). El hombre y la cultura. Buenos Aires: Sudamericana.

Boas, F. (1948). Cuestiones fundamentales de antropología cultural. Buenos Aires: Solar/Hachette.

Buchanan, E. (Ed.) (2004). Readings in Virtual Research Ethics: Issues and Controversies. Hershey, USA: IGI.

Cassirer, E. (1984). Antropología filosófica. México: Fondo de Cultura Económica.

Castells, M. (1998). La era de la información: economía, sociedad y cultura (Vol. 1. La sociedad red). Madrid: Alianza Editorial. 
Castro-Gómez \& Mendieta. (1998). Teorías sin disciplina. (latinoamericanismo, poscolonialidad y globalización en debate). México: Miguel Ángel Porrúa. Proyecto Hispánico. Recuperado el 20 de abril de 2008 de: http://www.ensayistas.org/ critica/teoria/castro/

Consalvo, M. \& Paasonen, S. (Eds.). (2002). Women and Everyday Uses of the Internet: Agency and Identity. New York: Peter Lang Publishing.

Constable, N. (2003). Romance on a Global Stage: Pen Pals, Virtual Ethnography, and "Mail-order" Marriages. Berkeley: University of California Press.

Crystal, D. (2002). El lenguaje e Internet. Madrid: Cambridge University Press.

De Certau, M. (1999). La cultura en plural. Buenos Aires: Nueva Visión.

Del Villar, D. (s.f.). Semiótica. Recuperado el 2 de mayo de 2008 de: http://www.monografias.com/ trabajos37/semiotica/semiotica.shtml

Deladalle, G. (1996). Leer a Peirce hoy. Madrid: Gedisa.

Deleuze, G. (1988). Diferencia y repetición. Madrid: Ediciones Jucar.

Dery, M. (1998). Velocidad de escape: la cibercultura en el final del siglo. Madrid: Editorial Ciruela.

Díaz, R. (1998). Archipiélago de rituales: teorías antropológicas del ritual. Barcelona: Anthropos. Universidad Autónoma Metropolitana.

Domínguez, F.D. (2006). Etnografía educativa más allá de la etnografía escolar. Trasvase de significados al ciberespacio. En Honorio M. Velasco Maillo, F. Javier García Castaño \& Ángel Díaz de Rada (editores). (2003). Lecturas de antropología para educadores. El ámbito de la antropología de la educación y de la etnografía escolar [26 párrafos]. Forum Qualitative Sozialforschung / Forum: Qualitative Social Research [On-line Journal], 7(2), Art. 2. Recuperado el 30 de agosto de 2007 de: http://www.qualitative-research.net/fqstexte/2-06/06-2-2-s.htm

Duch, L. (1998). Mito, interpretación y cultura: aproximación a la logomitica. Barcelona: Editorial Herder.

Durand, G. (2003). Mitos y sociedades: introducción a la mitología. Buenos Aires: Biblos.

Durkheim, E. (2003). Las formas elementales de la vida religiosa. Madrid: Alianza editorial.

E-14 Etnografías de lo digital. III Congreso de Cibersociedad. "Conocimiento abierto sociedad libre". Recuperado el 15 de mayo de 2007 de: http://www.cibersociedad.net/congres2006/gts/ gt.php?llengua=es\&id=117

Eliade, M. (1972). El mito del eterno retorno: arquetipos y repetición. Madrid: Guadarrama.

Eliade, M. (1973). Mito y realidad. Madrid: Guadarrama.

Eliade, M. (1974). Tratado de historia de las religiones. Madrid: Cristiandad.

Eliade, M. (1979). Lo sagrado y lo profano. Madrid: Guadarrama.

Faura, R. (2000). Cibercultura, irealidad o invención? 2 Congreso Virtual de Antropología y Arqueología, Octubre 2000. Recuperado el 20 de marzo de 2008 de: http://www.naya.org.ar/congreso2000/ ponencias/Ricard_Faura.htm

Foucault, M. (1971). Las palabras y las cosas. Una arqueología de las ciencias humanas. México: Siglo XXI Editores. 
Frazer, J. (1991). La rama dorada, magia y religión. México: Fondo de Cultura Económica.

Gadamer, H.G. (1977). Verdad y método (Vol. 1). Salamanca: Ediciones Sígueme.

García C., J. et al. (1994). Antropología de la educación. Madrid: Eudema.

García C., N. (1990). Culturas híbridas: estrategias para entrar y salir de la modernidad. México: Grijalbo.

García G., J. (1973). Antropología cultural y psicología. Madrid: Syntagma.

García, J.M. (2002). Virtualidad, realidad, comunidad. Un comentario sociológico sobre la semántica de las nuevas tecnologías digitales. Papers, 68 , 81-106.

García, M. (2005). Mediación tecnológica y nuevas formas de interacción social. En Tecnocultura y comunicación (pp. 176-188). Bogotá: Editorial Pontifica Universidad Javeriana.

Garrido, A. (2003). El aprendizaje como identidad de participación en la práctica de una comunidad virtual. UOC. Recuperado el 28 de julio de 2007 de: http://www.uoc.edu/in3/dt/20088/index.html

Geertz, C. (1997). La interpretación de las culturas: hacia una descripción densa. Barcelona: Editorial Gedisa.

Gibson, J. (1991). Celebration and Transgression: Nietszche on Ritual. Journal of Ritual studies, 5 (2).

Godelier, M. (1974). Economía, fetichismo y religión en las sociedades primitivas. Madrid: Siglo XXI.

Hamme, M. et al. (1994). Etnografía y métodos de investigación. Barcelona: Editorial Paidos.
Harris, M. (1985). El desarrollo de la teoría antropológica: una historia de las teorías de la cultura. México: Siglo XXI.

Heidegger, M. (1994). Construir, habitar, pensar (Eustaquio Barjau, traductor). Recuperado el 22 de abril de 2007 de: http://www.heideggeriana.com.ar/ textos/construir_habitar_pensar.htm

Heim, M. (1993).La ontología erótica del ciberespacio. En: Ciberespacio los primeros pasos. Consejo Nacional de Ciencia y tecnología. México: Editor Michael Benedikt.

Hine, C. (2004). Etnografía virtual. Barcelona: Editorial UOC.

Hjelmslev, L. (1971). Prolegómenos a una teoría del lenguaje. Madrid: Gredos.

Ibáñez, J. (1979). Más allá de la sociología. El grupo de discusión: técnica y crítica. Madrid: Editorial Siglo XXI.

Jones, S. (2003). Cibersociedad 2.0. Barcelona: Editorial UOC.

Lave J. \& Wenger, E. (2003). Comunities of Practice. Recuperado el 31 de julio de 2007 de: http://www. infed.org/biblio/communities_of_practice.htm.

Lavid, J. (2005). Lenguaje y nuevas tecnologías. Nuevas perspectivas, métodos y herramientas para el lingüista del siglo XXI. Madrid: Ediciones Cátedra.

Levy, P. (1999). ¿Que es lo virtual? México: Editorial Paidos.

López, A.C.; Seré, A. (editores). (2003). Nuevos géneros discursivos: los textos electrónicos. Madrid: Biblioteca Nueva, S.L.

Lorite, J. (Agosto de 1984). Rito y mito. Referentes estratégicos de comportamiento y legitimación del sistema interpretativo. Ideas y valores, 64-65. 
Magariños, J. (Abril-mayo de 2004). Los 4 signos diseño de las operaciones fundamentales en metodología semiótica. Razón y Palabra. Primera revista electrónica en América Latina especializada en comunicación, 38. Recuperado el 3 de mayo de 2008 de: http://www.razonypalabra.org. $\mathrm{mx} /$ anteriores/n38/.

Maldonado, T. (2007). Memoria y conocimiento: sobre los destinos del saber en la perspectiva digital. Barcelona: Gedisa Editorial.

Malinowski, B. (1948). Una teoría científica de la cultura y otros ensayos. Buenos Aires: Editorial Sudamericana.

Malinowski, B. (1974). Magia, ciencia, religión. BarceIona: Ariel.

Martí, D. (Abril-mayo de 2004). El análisis semiótico de hipertextos y la evaluación de websites. Razón y Palabra. Primera revista electrónica en América Latina especializada en comunicación, 38. Recuperado el 3 de mayo de 2008 de: http://www. razonypalabra.org.mx/anteriores/n38/.

Martí, D. (17 de enero de 2007). Internet desde las escuelas de los símbolos. Libro de Notas. Diario de los mejores contenidos de la Red en español. Recuperado el 3 de mayo de 2008 de: http:// librodenotas.com/opiniondivulgacion/10388/ internet-desde-las-escuelas-de-los-simbolos

Martín-Barbero, J. (2002). Oficio de cartógrafo. Santiago de Chile: Fondo de Cultura Económica.

Mayans I Planells, J. (2001). Recursos "virtuales" para el estudio de realidades "etéreas". El observatorio para la cibersociedad. Recuperado el 20 de noviembre de 2007 de: http://www.cibersociedad. net/x/extern.php?xtn=http: //www.ub.es/geocrit/ arac-65.htm
Mayans I Planells, J. (2002). Género chat o como la etnografía puso un pie en el ciberespacio. Barcelona: Editorial Gedisa.

Mayans I Planells, J. (2003a). De la incorrección normativa en los chats. Revista de investigación Lingüistica, $v$ (2), 101-116. Disponible en el archivo del Observatorio para la CiberSociedad. Recuperado el 30 de agosto de 2007 de: http://www.cibersociedad.net/archivo/articulo.php?art=43

Mayans I Planells, J. (2003b). Nuevas tecnologías, viejas etnografías. Objeto y método de la antropología del ciberespacio. Revista Quaderns de I'ICA, 17-18, 79-97. Disponible en el archivo del Observatorio para la CiberSociedad. Recuperado el 30 de agosto de 2007 de: http://www.cibersociedad.net/archivo/articulo.php?art=23

Mead, M. (1972). Educación y cultura. Buenos Aires: Paidos.

Miller, D. et al. (2000). The Internet: An Ethnographic Approach. Oxford: Berg.

Mitchell, W. (2003). The Cyborg Self and the Networked City. Cambridge, Mass: MIT Press.

Moraes, N. (Septiembre de 2005). Internet y ciberespacio en el estudio de comunidades diaspóricas: análisis de una experiencia. Comunicación presentada en el Simposio Antropología de los Media del X Congreso de Antropología organizado por la Federación de Asociaciones de Antropología del Estado Español. Recuperado el 25 de agosto de 2005 de: http://www.cibersociedad.net/archivo/ articulo.php?art $=208$

Noboa, M. (Abril-mayo de 2004). ¿Semiótica virtual para un diseño virtual? El diseño virtual a la luz de la semiótica. Razón y Palabra. Primera revista electrónica en América Latina especializada en comunicación, 38. Extraído el 3 de mayo de 2008 de: http://www.razonypalabra.org.mx/anteriores/ n38/. 
Ortiz, R. (1997). Mundialización y cultura. Buenos Aires: Alianza.

Pellerey, R. (Abril-mayo de 2004). Semiótica e informática: de 1948 a la actualidad. Razón y Palabra. Primera revista electrónica en América Latina especializada en comunicación, 38. Recuperado el 3 de mayo de 2008 de: http://www.razonypalabra. org.mx/anteriores/n38/

Pike, K. et al. (1985). Misionero y lingüista. En: M. Harris. (1985). El desarrollo de la teoría antropológica: Una historia de las teorías de la cultura. Madrid: Siglo XXI.

Rheingold, H. (1996). La comunidad virtual, una sociedad sin fronteras. Barcelona: Gedisa.

Rice, J. (2004). Writing About Cool: Hypertext and Cultural Studies in the Computer Classroom. Nueva York: Longman.

Rivas, M. (Febrero-abril de 2001). La semiosis: un modelo dinámico y formal de análisis del signo. Razón y Palabra. Primera revista electrónica en América Latina especializada en comunicación, 21. Recuperado el 3 de mayo de 2008 de: http:// www.razonypalabra.org.mx/anteriores/n21/21_ mrivas.html

Rodríguez, J.A. (1999). Hipertexto y literatura. Una batalla por el signo en tiempos posmodernos. Bogotá: Centro Editorial Javeriano.

Rodríguez, J.A. (2007). El relato digital. Hacia un nuevo arte narrativo. Bogotá: Editorial Libros de arena.

Sarlo, B. (1994). Escenas de la vida posmoderna; intelectuales, arte y videocultura. Madrid: Ariel.
Schaap, F. (2002). The Words That Took Us There: Ethnography in a Virtual Reality. Amsterdam: Askant Academic Publishers.

Serres, M. (1994). Atlas. Madrid: Cátedra.

Sorókina, T. (Junio de 2004). La semiótica, la escritura virtual y las permutas educacionales. Revista Iberoamericana de Educación, 33 (3). Recuperado el 25 de abril de 2008 de: http://www.rieoei.org/ deloslectores/681Sorokina.PDF.

Stone, A. (1993). ¿Podría ponerse de pie el cuerpo verdadero?: historias en el límite sobre las comunidades virtuales. En: Ciberespacio, los primeros pasos (pp. 81-113). México: Editor Michael Benedikt.

Strauss, L. (1987). Mito y significado. Madrid: Alianza.

Téllez, A. (2002). Nuevas etnografías y ciberespacio: reformulaciones metodológicas. Recuperado el 27 de noviembre de 2007 de: http://www. cibersociedad.net/congreso/comms/c10tellez. htm\#_edn1

Turkle, S. (1997). La vida en la pantalla. La construcción de la identidad en la era de Internet. Barcelona, Paidos.

Vasco, L. (Abril-mayo de 1987). Objetividad en Antropología: una trampa mortal. Uroboros, 1.

Velasco, H. et al. (1997). La lógica de la investigación etnográfica: un modelo de trabajo para etnógrafos de escuela. Madrid: Editorial Trotta.

Wallace, A. (1972). Cultura y personalidad. Buenos Aires: Paidós. 